\title{
Beyond the cholinergic crisis
}

\author{
Galle Medical Association Oration 2015
}

\section{Jayasinghe S S}

Department of Pharmacology, Faculty of Medicine, University of Ruhuna, Galle, Sri Lanka. South Asian Clinical Toxicology Research Collaboration, Department of Medicine, Faculty of Medicine, University of Peradeniya, Peradeniya, Sri Lanka.

Correspondence: Dr. Sudheera S Jayasinghe

e-mail: sudheerasj@yahoo.com

\begin{abstract}
Introduction: Organophosphates (OP) are the most frequently involved pesticides in acute poisoning. In Sri Lanka it has been ranked as the sixth or seventh leading cause of hospital deaths for many years. Neurotoxic effects of acute OP have been hitherto under-explored.

The aims of the studies were to assess the effects of acute OP poisoning on somatic, autonomic nerves, neuromuscular junction (NMJ), brain stem and cognitive function.
\end{abstract}

Methods: Patients following self-ingestion of OP were recruited to cohort studies to evaluated the function of somatic, autonomic nerves, NMJ, brain stem and cognition. Motor and sensory nerve function was tested with nerve conduction studies. Cardiovascular reflexes based autonomic function tests and sympathetic skin response (SSR) was used to evaluate autonomic function. NMJ function was assessed with slow repetitive supramaximal stimulation of the median nerve of the dominant upper limb. Brain stem function and cognitive function were assessed with Brain Stem Evoked Response Audiometry (BERA) and Mini Mental State Examination (MMSE) respectively. The data of the patients were compared with age, gender and occupation matched controls.

Results: There were 60-70 patients and equal number of controls in each study.

Motor nerve conduction velocity, amplitude and area of compound muscle action potential on distal stimulation, sensory nerve conduction velocity and F-wave occurrence were significantly reduced. At one week the significant impairment in autonomic function were change of diastolic blood pressure 3 min after standing, heart rate variation during deep breathing (HR-DB), SSR-amplitude and post-void urine volume. All except HR-DB were reversed at six weeks. No significant impairment of NMJ function, BERA and MMSE were noted.

Conclusions: Sub clinical somatic and autonomic nerve dysfunction was observed. There were no strong evidence of long term effects on NMJ, brain stem and higher function.

\section{Introduction}

Organophosphate (OP) compounds have become the most widely used pesticides for agricultural-pests throughout the world from the 1980s and the risk of acute and sub acute toxicity is high in humans (1). Acute pesticide poisoning is a major health problem especially in developing countries. It was estimated that one million serious, unintentional poisonings occurred and an additional two million people were hospitalized for attempted suicide with pesticides annually (2). In Sri Lanka, the majority of poisoning cases are self inflicted and $77 \%$ of the cases are in the age range of 11-30 years (3). OP and carbamate compounds were involved in $74 \%$ of pesticide poisoning (4).

OP bind to the esteratic site on the acetylcholine esterase (AChE) molecule, phosphorylates the enzyme, and lead to inhibition of its action (5). The binding between the esteratic site on the enzyme and the phosphorus atom is stable and takes hours or 
weeks to break off, depending on the compound involved. Studies have shown that a phenomenon of enzyme aging occurs which involves cleavage of a radical from the inhibited enzyme, making it resistant to reactivation. The net result is the accumulation of excess acetylcholine (ACh) at the cholinergic nerve endings all over the body resulting in the characteristic clinical manifestations. Following inhibition, recovery of this enzyme occurs at a rate of about one percent per day (5). Restoration of AChE levels occurs by spontaneous or induced reactivation of the enzyme and by new enzyme synthesis (5).

Accumulated acetylcholine at nerve endings initially stimulates and eventually leads to exhaustion of cholinergic synapses, resulting in neuromuscular junction (NMJ) dysfunction (5).

The problems occurring after OP poisoning have been identified to some extent. There are three well defined syndromes. These are acute cholinergic crisis, intermediate syndrome, organophosphate induced delayed polyneuropathy (OPIDN) and chronic organophosphate induced neuropsychiatric disorders (COPIND) (5).

Acute cholinergic crisis occurs due to accumulation of Ach, hence over activity of the nervous system. As a result the person with poisoning develops muscle twitching, narrowing of air ways, increased bronchial secretion, slowing of heart rate and low blood pressure. This cholinergic crisis is life threatening. Therefore the patient needs to be managed immediately to secure the life. Even though the patient is recovered from cholinergic crisis, the problems are not over.

Some patients develop muscle weakness, especially the proximal muscles within four days of ingestion. This entity is called the intermediate syndrome. The intermediate syndrome was first described and published by two Sri Lankans Prof. Nimal Senanayaka and Dr. Lakshman Karaliadda in 1987 and they coined the term intermediate syndrome. However problems are not yet over. There are things happening beyond the cholinergic crisis. OP can also cause polyneuropathy rarely. This occurs one to three weeks following poisoning. Patients complain of parasthesis and calf pain. Deep tendon jerks may be absent.

Therefore it is worth to look into what happens beyond the cholinergic crisis. This paper overview the effects of OP on nervous system including central and peripheral nervous systems. Further, it will describe the function of peripheral nervous system including somatic (motor and sensory), autonomic (sympathetic and parasympathetic), the junction between nerve and muscle, brain and higher functions of the brain.

\section{Methods}

Cohort studies were conducted with matched controls with the approval of the Ethical Review Committee, Faculty of Medicine, University of Ruhuna, Sri Lanka. Informed written consent was obtained from the patients and the controls. All clinical investigations were conducted according to the principles expressed in the Declaration of Helsinki.

The patients with self-ingestion of OP were recruited from a tertiary care hospital and a secondary care hospital in the Southern Province of Sri Lanka between June 2008 and September 2009. At the time of recruitment to the study, subjects either had features of the cholinergic syndrome or had been given atropine to counteract cholinergic syndrome in the peripheral units and then transferred to the collaborating hospitals.

OP poisoning was confirmed by the history from the patient and/or accompanying person, the cholinergic features and plasma cholinesterase activity $(\mathrm{ChE})$.

The controls were recruited from the persons accompanying the patients to the tertiary care hospital. Age, gender and occupation matched healthy volunteers who did not have a history of acute pesticide exposure were recruited within one month of the recruitment of the respective case. Age of the controls was matched to \pm 3 years of the patients.

Subjects with features of peripheral neuropathy, diabetes mellitus or those who were on long term medications, were excluded.

Motor nerve conduction studies (MNCS), sensory nerve conduction studies (SNCS), F-wave studies, autonomic function tests, slow repetitive nerve stimulation (RNS), brain stem auditory evoked response (BAER) and Mini Mental State Examination (MMSE) were performed at the time of discharge from the ward (the first assessment) and at six weeks (the second assessment) following 
acute exposure to OP. All neurophysiological investigations done on the patients were carried out on the controls. The room temperature of the neurophysiology laboratory was maintained at $25^{\circ} \mathrm{C}$.

The patients were assessed twice to explore the acute and subsequent effects on peripheral nerves. The earliest possible time to assess the patients was at the time of discharge and the earliest possible time for the second assessment with the least drop outs was at the sixth week.

The Neuropack MEB-9400A/K EMG/EP Measuring System (Nihon Koden) was used for electrophysiological assessment.

\section{SNCS}

SNCS were performed on median and ulnar nerves on both sides. The orthodromic method was used. Stimulating ring electrodes were placed on the second digit for median SNCS and on the fourth digit for ulnar SNCS. The ring cathode was placed around the digit near the metacarpophalangeal joint and the ring anode around the digit near the distal interphalangeal joint. A recording electrode was placed over the respective nerve on the anterior aspect of the wrist. Ground electrode was placed between the stimulating and the recording electrodes. The nerve was stimulated with supramaximal electrical stimulus.

Sensory nerve conduction velocities (SNCV) and the amplitude of the complex were recorded.

\section{MNCS}

MNCS were performed on median, ulnar and common peroneal nerves on both sides. Surface electrodes were used. The nerve was stimulated with supramaximal stimulation.

For the median MNCS, the recording electrode was placed over the abductor pollicis brevis, the reference electrode was placed over the proximal phalanx of the thumb and the ground electrode was attached between recording electrode and the stimulating probe. The stimulation was given on the palmar aspect of midwrist and at the elbow (antecubital fossa) just medial to the palpable brachial artery. The cathode was placed distal to the anode.
For the ulnar MNCS the recording electrode was placed on the mid portion of the abductor digiti quinti, the reference electrode was placed over the proximal phalanx of the fifth digit and the ground electrode was attached between recording electrode and the stimulating probe. The nerve was stimulated on the palmar aspect of wrist and just distal to the osseous groove in the posterior aspect of the medial epicondyle of the humerus, i.e., the ulnar groove.

For the common peroneal MNCS the recording electrode was placed over the extensor digitorum brevis muscle, the reference electrode was placed over the fifth toe and the ground electrode between the recording electrode and the stimulating probe. The nerve was stimulated on the lateral aspect of the popliteal fossa just medial to the insertion of the tendon of the biceps femoris and along the anterolateral surface of the fibula, 3 to $4 \mathrm{~cm}$ distal to the proximal tip of the fibular head.

Motor nerve conduction velocity (MNCV), amplitude and area of the compound muscle action potential (CMAP) on distal stimulation were recorded.

\section{F-wave studies}

F-wave studies were performed on median, ulnar and tibial nerves. Electrodes were placed as for MNCS on median and ulnar nerves. For the tibial F-wave studies, the recording, the reference and the ground electrodes were placed over the abductor hallucis muscle between the great toe and between the recording electrode and the stimulating probe respectively. The nerve was stimulated at a point slightly posterior and proximal to the medial malleolus. Sixteen stimulations were analyzed, percentage of F-wave occurrence and minimum reproducible F-wave latency were recorded.

\section{Autonomic function tests}

$\mathrm{R}-\mathrm{R}$ interval based autonomic function tests (heart rate response to standing, heart rate variation during deep breathing and heart rate response to Valsalva manoeuvre), blood pressure response to standing, blood pressure response to sustained handgrip, sympathetic skin response and residual urine volume were assessed. 


\section{Heart rate response to standing}

Participants were asked to stand up unaided from lying down position as quickly as possible when performing heart rate response to standing. The heart rate response was expressed by the 30:15 ratio; ratio of longest R-R interval around the $30^{\text {th }}$ beat after standing to shortest R-R interval around the $15^{\text {th }}$ beat after standing $(6,8)$.

\section{Heart rate response to deep breathing}

To perform heart rate variation during deep breathing, participants were asked to breathe deeply and evenly at 6 breaths per minutes ( 5 seconds in; 5 second out) for three cycles ( 30 seconds). Maximum and minimum R-R intervals were analyzed during each cycle and converted to beats per minute. Greatest heart rate differences (expiratoryinspiratory difference; E-I difference) during each cycle were calculated and the three differences averaged $(8,9)$.

\section{Heart rate response to Valsalva manoeuvre}

During the Valsalva manoeuvre, the participant breathed in to a mouth piece connected to a modified sphygmomanometer and maintained an expiratory pressure of $40 \mathrm{mmHg}$ for 15 seconds. The ratio of the longest R-R interval within 20 beats of ending the test to the shortest interval during the test was analyzed. The test was performed three times and the ratios from three Valsalva attempts averaged $(8,9)$.

\section{Blood pressure response to standing}

Blood pressure was measured while the participant was lying down quietly and around three minutes after standing (9) by using an OMRON SEM-1 automatic blood pressure monitor that uses the oscillometric method of blood pressure measurement. The monitor detects blood movement through the brachial artery and converts the movements into a digital reading.

\section{Blood pressure response to sustained handgrip}

In blood pressure response to sustained hand grip, the participant was asked to press a hand dynamometer with full strength. Then the hand grip was maintained at $30 \%$ of the maximum strength for five minutes.
Due to muscle contraction, sympathetic activity and vasoconstriction should lead to a rise in blood pressure. The diastolic blood pressure at the end of the effort should be at least $16 \mathrm{mmHg}$ higher than before the manoeuver. An increase in the diastolic blood pressure by $10 \mathrm{mmHg}$ or less is considered as abnormal $(8,9)$.

\section{Sympathetic skin response (SSR)}

Sympathetic skin response measures the skin potential which is evoked by stimulation (electric, auditory and visual). This potential shows the functioning of the sympathetic nerves of the sweat glands (10). Participant's skin was cleaned where the electrodes were attached to remove any moisture and gel from the skin. The active electrode was pasted on the center of the palm of the dominant hand. The reference electrode was pasted on the center of the back of the same hand. The ground electrode was attached between the active and the stimulating electrodes. The stimulating electrode was placed on the median nerve of the recording wrist and stimulated with the intensity of $25 \mathrm{~mA}$. Six stimulations were administered with at least $30 \mathrm{sec}$ intervals between each stimulation. The latency was measured from the onset of stimulus artifact to the onset of negative deflection. The amplitude was measured from the baseline to negative or positive peak whichever was the highest. Average latency and maximum amplitude were recorded.

\section{Residual urine volume}

Post void residual urine volume was measured ultrasonically after a voluntary void $(11,12)$.

\section{Exercise modified slow repetitive supramaximal stimulation}

Exercise modified slow repetitive supramaximal stimulation (RNS) was used to assess NMJ function on the median nerve of the dominant upper limb. RNS was carried out administering 10 stimulations at a rate of $3 \mathrm{~Hz}$ with a filter setting of $20 \mathrm{~Hz}-5 \mathrm{kHz}$. Forearm and hand were fixed by straps to restrict movements and to ensure that contraction of the abductor pollicis brevis was isometric. Recording electrodes were placed over the abducter pollicis brevis, the reference electrode was pasted over the 
proximal phalanx of the thumb and the ground electrode was attached between the recording electrode and the stimulating probe (13). Laboratory temperature was maintained at $25^{\circ} \mathrm{C}$ during the test.

The nerve was stimulated at the wrist at rest- the first train (A), immediately after 30 seconds maximal isometric exercise - the second train (B), and two minutes after the exercise - the third train (C) (14). Participants were asked to abduct the thumb against fixed resistance for the isometric exercise (14). The post exercise facilitation and the post exercise exhaustion were calculated after the isometric exercise $(13,14)$.

The decrement responses were quantified by calculating the percentage change in amplitude as follows: (15)

\section{- At rest - the first train of stimuli(A)}

- percentage change in amplitude between the second and the first action potential of the first train of stimuli $\left(\left(\mathrm{A}_{1}-\mathrm{A}_{2}\right) / \mathrm{A}_{1} \times 100\right)$

- percentage change in amplitude between the fourth and the first action potential of the first train of stimuli $\left(\left(\mathrm{A}_{1}-\mathrm{A}_{4}\right) / \mathrm{A}_{1} \times 100\right)$

- percentage change in amplitude between the fifth and the first action potential of the first train of stimuli $\left(\left(\mathrm{A}_{1}-\mathrm{A}_{5}\right) / \mathrm{A}_{1} \times 100\right)$

- Immediately after 30 seconds maximal isometric exercise - the second train of stimuli (B)

- percentage change in amplitude between the second and the first action potential of the second train of stimuli $\left(\left(\mathrm{B}_{1}-\mathrm{B}_{2}\right) / \mathrm{B}_{1} \times 100\right)$

- percentage change in amplitude between the fourth and the first action potential of the second train of stimuli $\left(\left(\mathrm{B}_{1}-\mathrm{B}_{4}\right) / \mathrm{B}_{1} \times 100\right)$

- percentage change in amplitude between the fifth and the first action potential of the second train of stimuli $\left(\left(\mathrm{B}_{1}-\mathrm{B}_{5}\right) / \mathrm{B}_{1} \times 100\right)$

- Two minutes after the exercise - the third train of stimuli (C)

- percentage change in amplitude between the second and the first action potential of the third train of stimuli $\left(\left(\mathrm{C}_{1}-\mathrm{C}_{2}\right) / \mathrm{C}_{1} \times 100\right)$
- percentage change in amplitude between the fourth and the first action potential of the third train of stimuli $\left(\left(\mathrm{C}_{1}-\mathrm{C}_{4}\right) / \mathrm{C}_{1} \times 100\right)$

- percentage change in amplitude between the fifth and the first action potential of the third train of stimuli $\left(\left(\mathrm{C}_{1}-\mathrm{C}_{5}\right) / \mathrm{C}_{1} \times 100\right)$

A decline of compound muscle action potential (CMAP) amplitude up to $8 \%$ with RNS is considered as normal (15).

The post exercise facilitation was assessed by calculating the ratio of the amplitude of the first action potential in the second train to the amplitude of the first action potential of the first train $\left(B_{1} / A_{1}\right)$ $(13,14)$ and the post exercise exhaustion by calculating the ratio of the amplitude of the first action potential of the third train to the amplitude of the first action potential of the first train $\left(\mathrm{C}_{1} / \mathrm{A}_{1}\right)(14)$.

\section{Brain Stem Auditory Evoked Response (BAER)}

BAER is an electrophysiological method that can be used for neurotoxicity testing of auditory function (16). A computer based Neuropack $\mathrm{S}_{1}$ EMG/EP measuring system MEB-9400 (Nihon Kohden) was used for the BAER. A piece of cotton moistened with alcohol was used to clean the patient's skin where the electrodes were attached and the skin rubbed with dry gauze to remove any moisture and gel from the skin. Recording electrodes (active and reference) and grounding electrodes were attached on the patient's head with Elefix paste. The patient was kept on a chair relaxed with eyes closed. To avoid EMG of the neck, patients were asked not to move their head. To avoid EMG of the chin, patient was asked to open his / her mouth slightly. The headphones were kept on the head. The hi-cut and the low-cut filters were set to $3 \mathrm{kHz}$ and $100 \mathrm{~Hz}$ respectively. The skinelectrode contact impedance was kept below $5 \mathrm{k} \Omega$. The stimulation intensity was $90 \mathrm{~dB}$ and 1000 stimulations were averaged. Latency was measured in msec. Interpeak latencies of I-III, III-V and I-V were measured $(16,17)$.

\section{Mini Mental State Examination}

Cognitive function was assessed only if the educational level of the participant was at least up to year five. People who were suffering from dementia, psychiatric illness and those on medication which 
have the potential to alter cognitive function (anticonvulsants; vigabatrin, gabapentin and lamotrigine, minor tranquilizers; diazepam, and antihypertensive like methyldopa) were excluded from the study. Cognitive function was assessed once the patient had recovered from the acute cholinergic crisis.

A validated Sinhalese translation of the MMSE was used with a few modifications (18). Table 1 illustrates the modifications made in the MMSE administered in our study which reflected our need to use this in the community. A total score of less than 24 out of 30 was considered as cognitive impairment. No response or refusal to perform any item was scored as zero.

\section{Estimation of plasma $\mathrm{ChE}$ activity}

Generally the term acetylcholinesterase activity is referred to red blood cell acetylcholinesterase or acetylcholinesterase at the nerve tissue. However we analyzed $\mathrm{ChE}(\mathrm{ChE}=$ acetylcholinesterase $(\mathrm{AChE})$ plus butyrylcholinesterase (BChE)) activity in plasma. The modified Ellman method developed by Worek F et al. (1999) was used to estimate ChE activity in plasma (19). Plasma samples were obtained from EDTA blood after centrifugation (10 $\mathrm{min}, 500^{*} \mathrm{~g}$ ) and stored in $1 \mathrm{ml}$ aliquots at $-80^{\circ} \mathrm{C}$ until analysis.

Prior to analysis the thawed samples were kept on ice until analysis. Preparation of inhibited cholinesterase were made by incubating plasma samples with PX-ethyl, PX-methyl and obidoxime for $15 \mathrm{~min}$ at $37^{\circ} \mathrm{C}$ followed by immediate dilution of the samples (1:100 in diluting reagent) and freezing.

The activity of cholinesterase was measured with a thermostatted filter photometer (Model $1101 \mathrm{M}$, Eppendorf, Hamburg, Germany) at $436 \mathrm{~nm}$ and $37^{\circ} \mathrm{C}$ using polystyrol cuvets.

Cholinesterase enzyme activity was calculated by using the equation,

$\operatorname{Activity}(\mu \mathrm{mol} / \mathrm{l} / \mathrm{min})=\frac{\operatorname{Sample}(\mathrm{mE} / \mathrm{min})-\operatorname{Blank}(\mathrm{mE} / \mathrm{min})}{10.6}$

The analysis was done at Walther Straub Institute for Pharmacology and Toxicology, Munich, Germany.

\section{Statistical analysis}

Graph Pad Prism 4 and Social Package of Statistical Software were used for the statistical analysis. Normal distribution of the data was tested with Kolmogorov-Smirnov test. Data which had no normal distribution were analyzed with nonparametric tests. The paired T-test was used to compare the results of the first and the second assessment and the unpaired T-test was used to compare the results of the patients and the controls. Correlation of neurophysiological indices with potential confounders was analyzed with Spearman's correlations. Multiple liner regression model was used to adjust for potential confounders.

\section{Results}

From a total of 163 acute OP poisoning admissions to the collaborating hospitals, $60-70$ patients participated for each study. Mean (SD) GCS on admission was 14 (2). All patients received atropine, 54 patients received pralidoxime.

Plasma ChE activity at four and/or twelve hours after the exposure was available in 33 patients. The median (inter quartile range) of plasma ChE activity at four and twelve hours was 790 (146 - 2598) $\mu \mathrm{mol} / \mathrm{l} / \mathrm{min}$ and $431(136-3068) \mu \mathrm{mol} / \mathrm{l} / \mathrm{min}$ respectively.

None of the patients or the controls had diabetes mellitus. The mean $\mathrm{HbA}_{1} \mathrm{C}$ of patients and the controls were $5.4 \pm 0.5 \%$ and $5.7 \pm 0.6 \%$.

\section{Function of peripheral nerves}

The neurophysiological assessments and the results of SNCS, MNCS and F-wave studies are shown in the Table 1.

Impairment of peripheral nerve function was observed at both occasions in the cases compared to the controls. In the first assessment these were significant for MNCV of median, ulnar and common peroneal nerves, SNCV and CMAP amplitude of ulnar nerve and $\mathrm{F}$ wave occurrence of median, ulnar and tibial nerves. However no abnormality was detected when compared to the standard cut-off values for normal MNCS and SNCS except F-wave occurrence. 


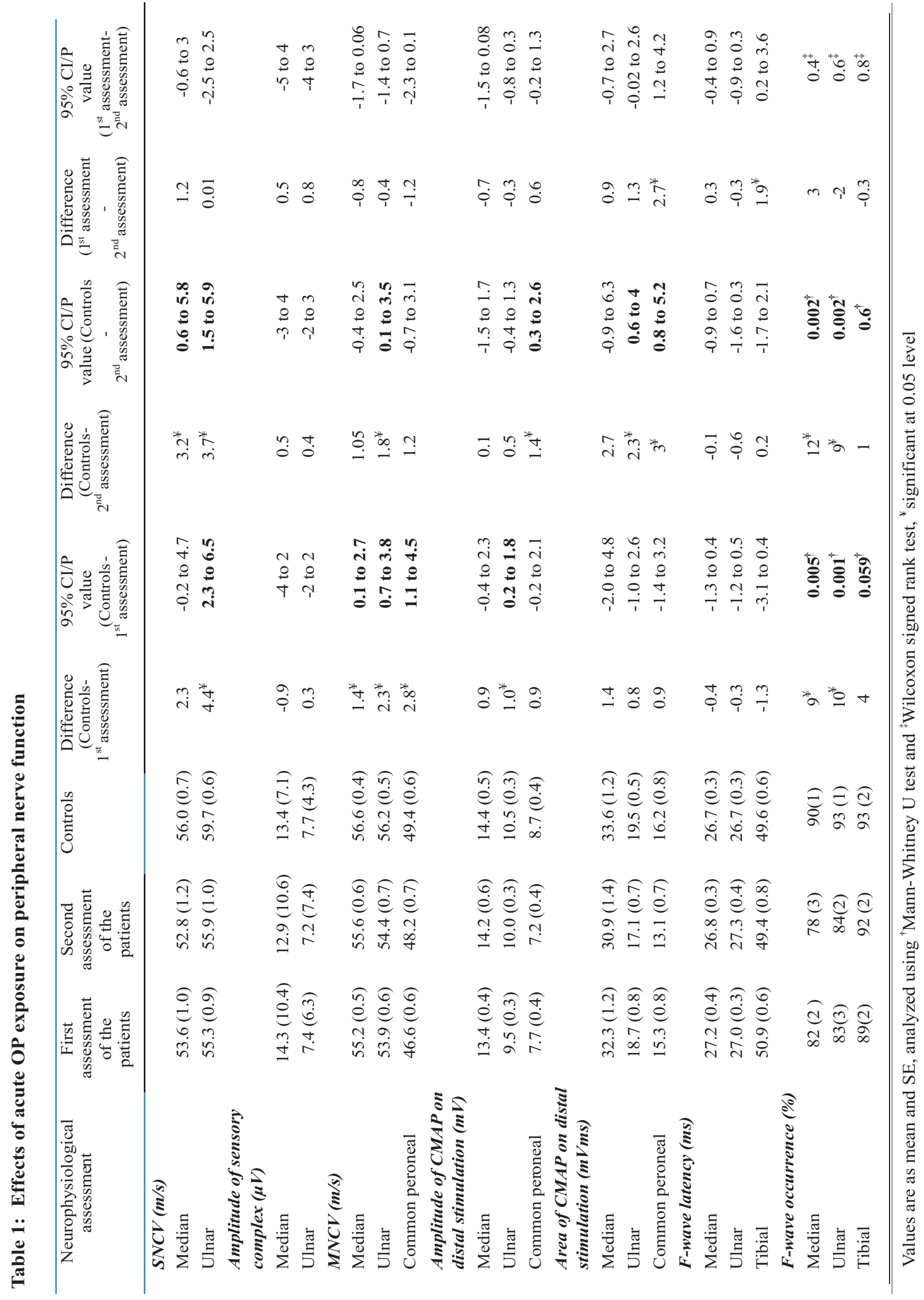


In the second assessment significant worsening of peripheral nerve function was seen in common peroneal CMAP-area and reduction of tibial F-wave latency compared to the first assessment. MNCV of median and common peroneal nerves, amplitude of ulnar nerve CMAP on distal stimulation and F-wave occurrence of tibial nerve reversed to normal at the second assessment.

Seventeen patients were admitted to Intensive Care Unit. Among them seven patients died. Four of the $10(40 \%)$ survivors underwent neurophysiological assessment at median (range) of 24 (6-40) days after the exposure.

Correlation of neurophysiological indices with potential confounders was looked into. None of the neurophysiological indices significantly correlate with plasma ChE activity. However MNCV of ulnar; SNCV of median and ulnar, MNCV of ulnar; MNCV of median, ulnar and tibial F-wave occurrence showed significant negative Spearman's correlations with smoking habits, PAM therapy and alcohol consumption respectively.

When multiple liner regression models were used to adjust for potential confounders, effects on SNCV of median nerve, area of CMAP of median nerve and F-wave latency of tibial nerve with prlidoxime therapy and the area of CMAP of ulnar nerve with the type of OP ingested showed statistical significance.

To determine whether any multicollinearity were present, and to understand whether there is a strong linear association between each predictor variable and all other remaining predictors, the Variance Inflation Factor (VIF) and condition indices were examined. None of the VIF exceeds 10 and a condition index exceeds $30(20)$.

\section{Function of autonomic nerves}

Statistically significant autonomic dysfunction was seen in the first assessment of the patients compared to the controls in change of diastolic blood pressure (DBP) 3 minutes after standing, HR-DB, SSRamplitude, SSR-latency and post-void urine volume (Table 2). At six weeks recovery of autonomic dysfunction was observed except in HR-DB (Table 2).
When the number of patients with abnormal autonomic function test was compared with the number of controls with abnormal autonomic function tests (by Chi-Square test) in relation to standard cut-off values of autonomic function tests, no statistically significant difference was observed.

Sixteen patients were not able to perform blood pressure response to sustained hand grip due to painful cannula site on their dominant hand. Twentyfour patients were not able to complete the test. Twenty-one patients in the second assessment and 15 controls were also not able to complete the test. Participants (26 in the first assessment, 30 in the second assessment and 55 in the controls) who could complete the test were within the normal limits. However odds ratio of the number of patients who were able to complete the test in the first assessment (vs controls) and the second assessment (vs controls) were 3.4 and 2.3 respectively.

The complex of SSR was almost flat in seven patients in the first assessment and in one patient in the second assessment. None of the controls showed flat SSR complex.

The urinary bladder of most patients was catheterized. The catheter was removed only at the time of discharge from the hospital. Hence residual urine volume was measured in 27 patients on discharge and 24 matched controls (Table 2).

In the correlation matrix, it was observed that none of the bivariate correlations are highly correlated except alcohol consumption and smoking habits, GCS on admission and smoking habits. To determine whether any multicollinearity were present, and to understand whether there is a strong linear association between each predictor variable and all other remaining predictors, the Variance Inflation Factor (VIF) was examined. None of the VIF exceeds 10 .

Adjusted multiple linear regression revealed significant effects of alcohol consumption on change of SBP 3 min after standing $(\mathrm{B}=4.4, \mathrm{SE}=$ $1.6, \mathrm{p}=0.009)$ and size of the pupil $(\mathrm{B}=-0.6, \mathrm{SE}=$ $0.3, \mathrm{p}=0.04)$; and the effects of smoking on residual urine volume $(\mathrm{B}=11.8, \mathrm{SE}=5.1, \mathrm{p}=0.03)$ and size of the pupil $(\mathrm{B}=0.5, \mathrm{SE}=0.2, \mathrm{p}=0.04)(21)$. 


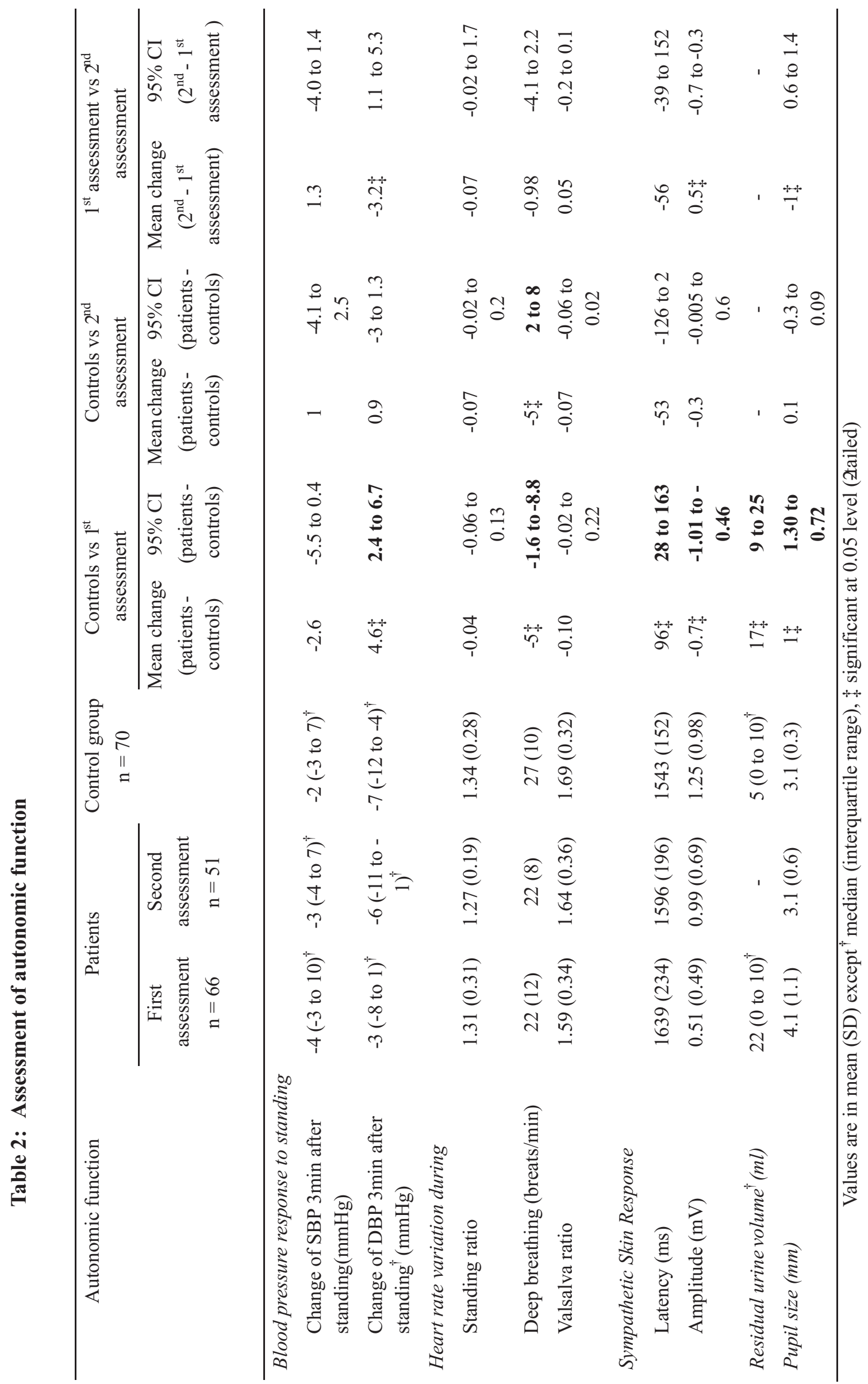




\section{Slow repetitive nerve stimulation}

Table 3 shows decrement response at rest, immediately after isometric exercise, two minutes after the exercise, post exercise facilitation and post exercise exhaustion. Statistically significant difference of decrement response was observed at rest $\left(\mathrm{A}_{4}\right)$ and two minutes after the exercise $\left(\mathrm{C}_{4}, \mathrm{C}_{5}\right)$ in the second assessment compared to the controls.

The decrement response was significant at rest $\left(\mathrm{A}_{4}, \mathrm{~A}_{5}\right)$ and two minutes after the exercise $\left(\mathrm{C}_{4}\right)$ in the second assessment compared to the first assessment.

Seven occasions in the patients in the first assessment and five occasions in the controls showed more than $8 \%$ decrement response either to the second, fourth or fifth stimuli.

\section{BAER}

The differences of the mean latency of I - III, III - V and $\mathrm{I}-\mathrm{V}$ were prolonged in the test group compared to the controls except in right side I - III difference in the first assessment of the test, right side I - III and $\mathrm{I}-\mathrm{V}$ difference in the first and the second assessment in the test group compared to the controls. None of the differences were statistically significant (Table 4) (22).

\section{MMSE}

There were 60 patients and 61 controls. The mean age (SD) of the patients and controls were 31.5 (11.6) years and 31.3 (11.8) years respectively. Forty-two patients turned-up for the follow up assessment at six weeks following the exposure. Three out of 60 patients required Intensive Care Unit (ICU) admission.

Seventeen out of 60 patients in the first assessment and 9/61 in the controls scored less than 24 in the $\operatorname{MMSE}\left(\mathrm{X}^{2}=3.3, \mathrm{p}=0.06\right)$. Seven patients out of 42 showed MMSE score less than 24 in the second assessment. There was no significant difference between the number of patients with impaired MMSE in the second assessment vs controls $\left(\mathrm{X}^{2}=\right.$ $0.07, p=0.7)$ and the second assessment vs the first assessment $\left(\mathrm{X}^{2}=1.8, \mathrm{p}=0.17\right)$. All three patients who had ICU admissions had MMSE score more than 26 .
Table 5 indicates mean (SD) score of individual domains of MMSE in the patients and the controls. The total score of MMSE, orientation and the language domains of MMSE showed significant impairment in the first assessment of the patients compared to the controls. There was no significant impairment when scores in the second assessment was compared with the controls (23).

\section{Discussion}

Small magnitude adverse difference of SNCV, MNCV, amplitude and area of CMAP on distal stimulation and F-wave occurrence in acute $\mathrm{OP}$ poisoned patients compared to the controls was observed. Although it is a small magnitude, the difference is clinically significant as any damage to be evident by electro physiologically nearly $50 \%$ of the fibers need to be damaged as the remaining unaffected fibers compensate the affected ones. Therefore, the absolute values are greatly underestimated.

The results of autonomic function tests showed significant impairment of heart rate variation during deep breathing and the patients had four fold residual urine in their bladder compared to the healthy subjects.

There was no detectable NMJ dysfunction assessed with exercise modified slow RNS or significant difference in the inter peak latencies of BAER after clinical recovery of acute OP poisoning.

Although there was a slight transient cognitive impairment detected with the screening tests following acute OP exposure, no long term cognitive defects was detected by MMSE in clinical settings.

Diabetes is well known to cause neuropathy. Therefore we excluded patients with diabetes from the study. Occupation matched controls were recruited since there is evidence that occupational exposure to pesticides can cause neuropathies. Very few studies were found in the literature which focused on SNCS / MNCS following acute ingestion of OP. There have been no studies which have looked at the effects of acute OP exposure on peripheral nerve function compared to matched controls. Most large studies have examined nerve function in farm workers who had chronic, probably low level exposure to pesticides. 


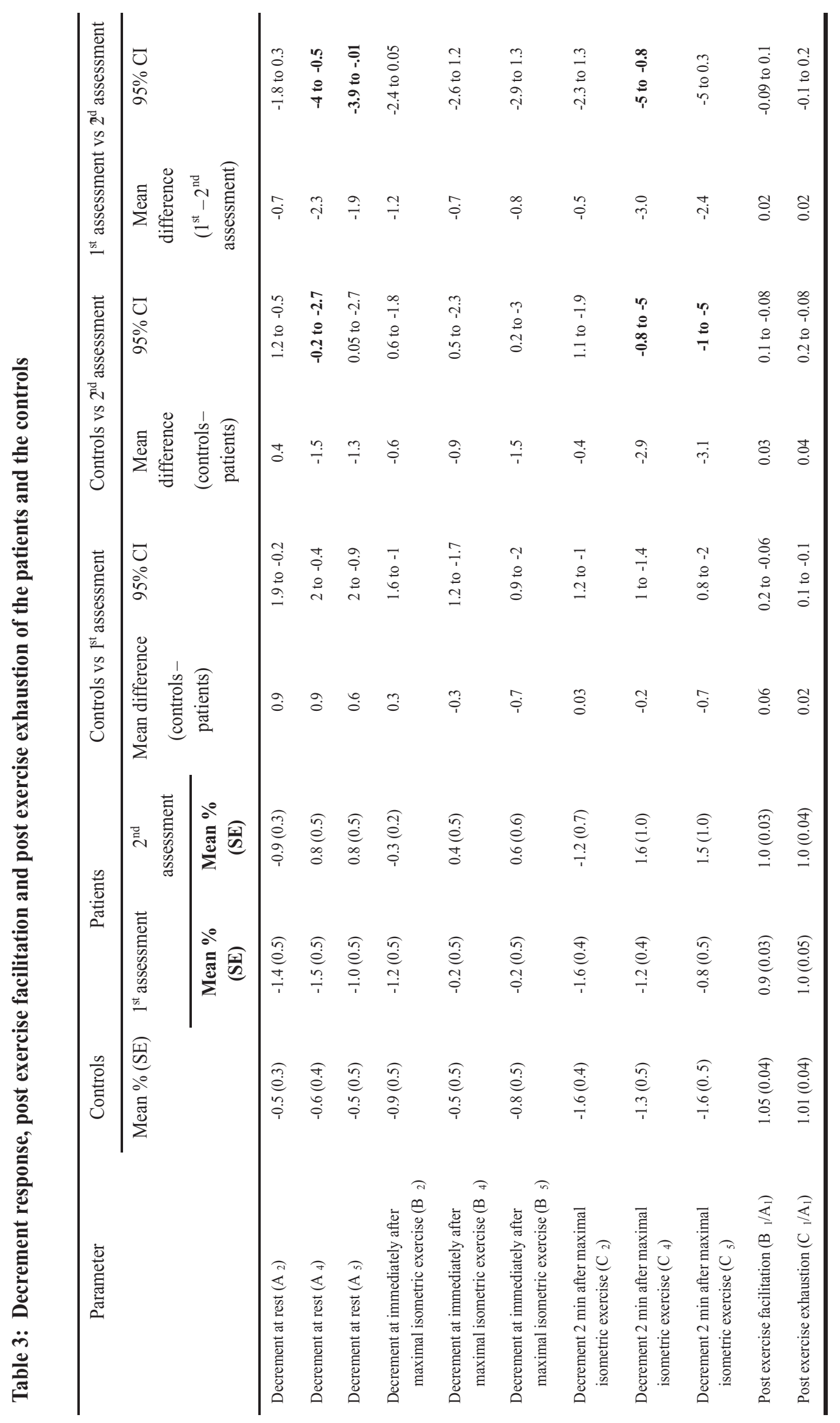




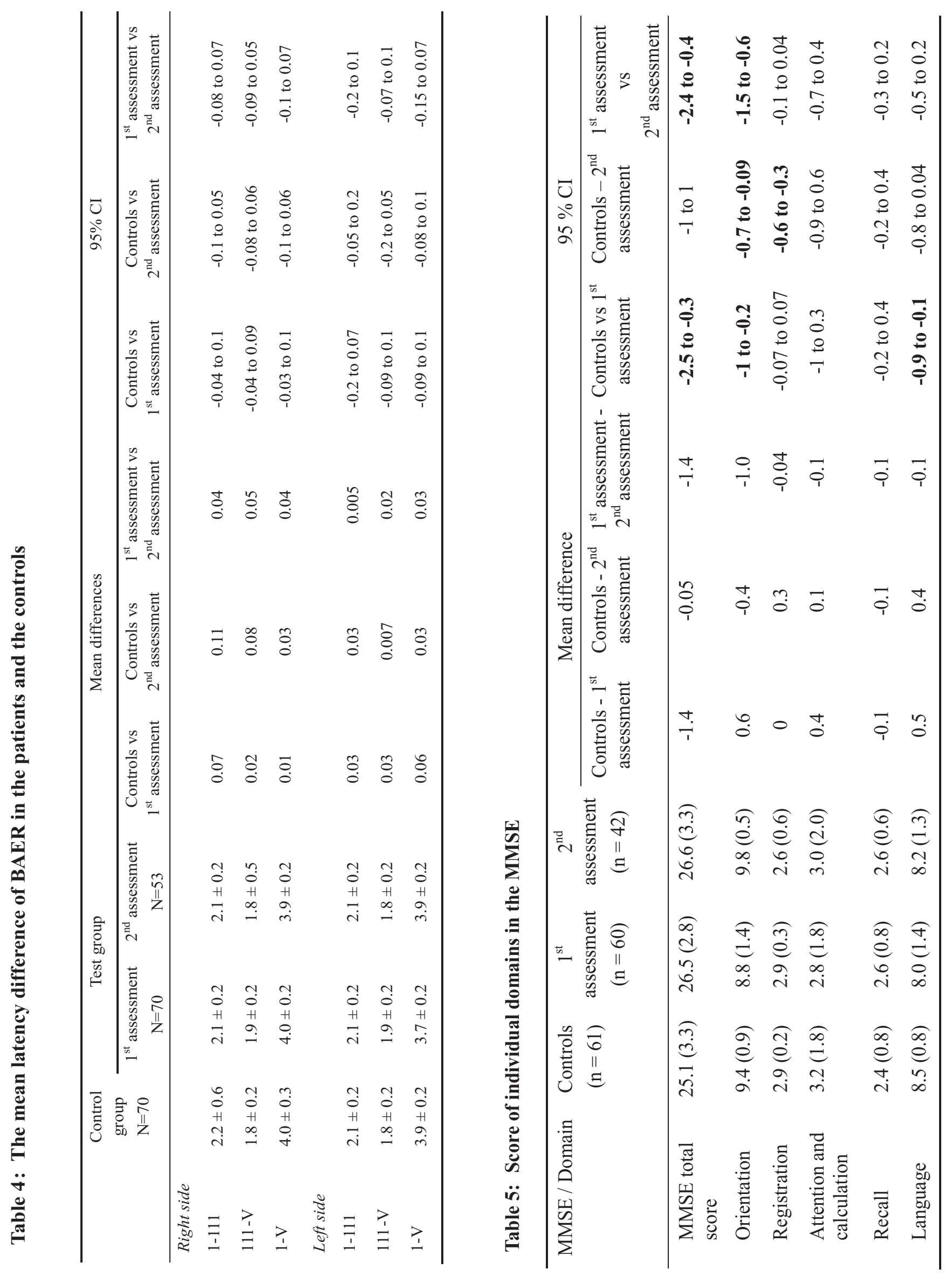


Reduction of SNCV and MNCV of the patients in our study indicate that there may be demyelination following acute OP exposure. Since we did not follow up the patients beyond six weeks of exposure we do not know whether the changes were reversed or worsened with time.

Reduced amplitude and/or area of CMAP on distal stimulation were observed in several comparisons. These indicate that there may be an axonal damage since amplitude and area under negative curve of CMAP are directly proportional to the number of functioning axons (10). If the whole length of the nerve is affected, F-wave latency should be prolonged. Reduced nerve conduction velocity only in the distal segment may be an evidence of distal demyelination with sparing of proximal segment. Since we did not perform segmental nerve conduction studies focal damage cannot be excluded.

F-wave studies are also important for recognizing the proximal segment involvement since it is produced by antidromic activation of motor neurons. It is also important to identify marginal changes of standard MNCS since an impulse travels a long pathway to produce an $\mathrm{F}$-wave, and additive effects are more obvious in F-wave latency measurement as it measures the delay in a much longer segment (10). The latency of and F-wave includes the time required for the evoked action potential to ascend antidromically to the anterior horn cells, the time between the beginning of antidromic activation and subsequent orthodromic discharge (the central "turnaround" time) and the time required for the resultant action potential to descend orthodromically from the anterior horn cells to the muscle fibers. F-wave abnormalities may be loss of response (reduction of occurrence) or prolonged latencies (10). F-waves do not occur with each stimulation. They depend on excitability of motor neurons. In normal individuals $\mathrm{F}$-wave occurrence is more than $90 \%(10)$.

There was a statistically significant reduction of F-wave occurrence observed in the median and the ulnar nerves in the first assessment compared to the controls. Although there was prolongation of F-wave latency, it was not statistically significant.

Some nerves such as the ulnar and the common peroneal are highly vulnerable to external damage in the ulnar groove and behind the head of the fibula respectively. Improper nursing care may be partially contributed to the nerve damage.

Although autonomic function in farm workers has been looked in to, there were no studies that looked at autonomic function following acute OP exposure. In the current study neurophysiological parameters of the exposed individuals were compared with the parameters of matched controls.

Variation of heart rate during rest, deep breathing and isometric muscle contraction were tested in flower bulb farmers who had chronic mixed pesticide exposure by Ruijten M.W.M.M et al. (1994). Significant autonomic dysfunction was shown in variation of heart rate during rest and deep breathing (24). We did not assess variation of heart rate during rest but results for heart rate variation during deep breathing were similar in both studies .

In the tests reflecting parasympathetic damage (heart rate response to Valsalva manoeuver, heart rate variation during deep breathing and immediate heart rate response to standing) a positive result was shown only with heart rate response to deep breathing. It is well known that the response of the heart rate can be abolished by atropine (7). The first assessment of the patients was conducted within the mean of three (IQR 1 - 4) days after the cessation of atropine therapy. The effects of atropine may not have disappeared completely even 6 weeks after exposure. On the other hand, only 48/66 patients were able to do the Valsalva manoeuver. The sample size may not have been adequate to draw a conclusion. Or else the autonomic function of exposed individuals may not have been affected.

Change of diastolic BP 3 min after standing was significantly low in the patients, but recovered completely at six weeks after exposure. Blood pressure response to standing reflects sympathetic function. But it begins to show abnormal results with more severe sympathetic nerve damage (7). Sympathetic damage in our patients may not have been severe enough to give abnormal results in the blood pressure response to standing.

Reduction of amplitude and prolongation of latency in SSR are in favor of sympathetic damage at one week after exposure. SSR represents the change in voltage measured at the skin surface following a single electrical stimulus. It depends on the electrical activity arising from sweat glands (10). 
Latency of SSR is inversely proportional to the number of sweat glands innervated (10). Hence sympathetic innervations to sweat glands were less in the patients compared to the controls. In the second assessment both the latency and the amplitude of SSR were improved. We could not eliminate the effects of atropine on sweat glands and changes in the first assessment may have been due to effects of atropine. However patients did not show sympathetic dysfunction as reflected in SSR at six weeks after exposure.

It is well known that high residual urine volume is associated with increased risk of urinary tract infections. Since our results showed that residual urine volumes in patients were significantly higher than in the controls, it is very important to follow up such patients further.

One third of patients were admitted with coingestion of alcohol. Acute alcohol ingestion is unlikely to have had any effects on the parameters we assessed, since the assessments were carried out at one week after the exposure. It is well known that long term alcohol consumption is associated with neuropathies including autonomic neuropathy. Adjusted multiple linear regression did not show widespread effects of alcohol consumption on autonomic function.

The number of patients who could complete the test of blood pressure response to sustained hand grip was significantly lower than the number of controls who could complete the test. The difference may be due to muscle weakness following poisoning.

The gold standard electrophysiological tool to explore the neuromuscular junction function is single-fiber electro myography (SF-EMG) $(15,25$, 26). We explored whether RNS with exercise might be a useful alternative method as SF-EMG is not available in most centers.

Few patients had RNS abnormalities in the first assessment and this was no different from the controls. Results of RNS six weeks after the exposure showed significant decrement response. However RNS does not appear to be a useful method to explore medium to long term NMJ function.

NMJ dysfunction has been demonstrated by fast repetitive nerve stimulation with $\mathrm{OP}$ poisoning before clinical recovery (27), but there is no evidence whether these changes persist over the long term.
A study done to see the effects of occupational exposure to OP on neuromuscular function did not show any detectable neuromuscular disturbances with RNS and with SF-EMG (28).

The frequency of neuromuscular transmission abnormalities detected by SF-EMG was significantly higher than those detected by RNS $(28,29)$.

Decrement response is more obvious in fast repetitive stimulation than the slow repetitive stimulation. The current study concentrated only the slow repetitive stimulation with exercise modification to magnify the function of NMJ. However this may not be sensitive enough to detect subtle abnormalities (30).

The amplitude of compound muscle action potential may increase during high-frequency nerve stimulation or after voluntary activation of muscle (10). The measurement of CMAP amplitude in fast repetitive stimulation is not recommended by some since amplitude of the CMAP during high frequency stimulation may give variable and inconsistent results (15). Therefore we measured CMAP amplitude just after and two minutes after the isometric muscle exercise. Even with isometric muscle exercise the current study was not able to demonstrate significant decrement comparing to the controls. Previous studies on neuromuscular synapse function in organophosphate workers also did not show significant decrement response compared to the controls $(13,14)$.

With each depolarization, $\mathrm{Ca}^{++}$is release into the periterminal space. If another depolarization occurs, high $\mathrm{Ca}^{++}$concentration leads to increase in acetylcholine quanta released. If neuromuscular transmission is impaired this greater acetylcholine release briefly improves synaptic transmission, producing facilitation (15). Marked facilitation is characteristic of presynaptic blockade. Although facilitation can also sometimes be positive with postsynaptic problems (15).

Although it was recommended to calculate the changes in CMAP size between the first and the fourth or the fifth response of a train of stimuli (15) we took the second response as well, since Maselli et al (2004) and Jayawardana et al (2008) observed the maximum decrement at the second stimulus $(27,31)$. The data set of the current study showed 
the maximum decrement either at the second or the third stimulus.

The majority of the cases of the current study showed increment pattern progressively over the train. For that reason the mean percentage decrements shown in the table 2 are negative values. This pattern is described as pseudo increment and is a normal variant (32).

Even though a decrement response $(>8 \%)$ was observed in few occasions both in the patients and the controls, even these decrements were not confirmed. False positive results of repetitive nerve stimulation is possible since the studies are technically difficult; artifacts resulting from electrode placement, movements, changes of skin resistance and intramuscular temperature cannot be entirely controlled (25).

Very few human studies have examined ototoxic effects with pesticide exposure. No studies have assessed the ototoxic effects following acute exposure to OP.

BAER is a sensory evoked potential used to assess the integrity of the auditory pathway from the eighth nerve to the auditory cortex (16). The inter peak latencies (I - III, III - V and I - V) of BAER provide a straightforward method to roughly localize lesions in the auditory pathway (16).

Animal studies by Bielefeld E C et al. (2005) and Harris K C et al. (2006) showed shifting of evoked potential threshold and loss of inner and outer hair cells following application of $10 \mathrm{mM}, 5 \mathrm{mM}$ and $3 \mathrm{mM}$ of PQ to cochlea through a surgical incision $(33,34)$. The current study did not look into the auditory threshold level.

Clerici W J (1996) demonstrated disruption of generation and transmission of afferent cochlear signals by generation of reactive oxygen species within the perilymphatic space with artificial perilymph and hydrogen peroxide (35). Even though OP produces reactive oxygen species after ingestion, the concentration of substances reaching the perilymphatic space may not be sufficient to damage the cochlea.

Kimura K et al. (2005) studied the effects of the event-related evoked potentials in tobacco farmers who were exposed to different pesticides including OP. They did not see any significant effects on the event-related evoked potentials (36).
Cognitive function was tested with the simple and quick assessment tool of MMSE. The MMSE provides a measure of cognition which covers a broad set of cognitive domains: orientation, registration, short term memory, attention, calculation, visuo-spatial skills and apraxia (18). The sensitivity and specificity of MMSE were $100 \%$ and $69 \%$ to the cut off level of less than 24 evaluated against the performances at CAMCOG (Cambridge Cognitive Score) (18).

Stephens R et al (1995) reported impaired cognitive function in sheep farmers who were exposed to OP through sheep dipping, which involves immersing each animal in a pesticide solution to control parasitic infections. Exposure to OP was appeared to be splashing on to the skin where protective cloths were seldom worn. The speed of performance of simple reaction time, symbol-digit substitution and syntactic reasoning were significantly slower in the farmers than the controls. No impairment was found in short term memory (by digit span test and visual spatial memory) and long term memory (by serial word learning, category search classification, category search recognitions) in the cases compared to the controls $(1,37)$.

Steenland K et al (1994) investigated chronic squeal of acute occupational OP poisoning $(38,39)$. They studied cognitive function via finger tapping (motor speed test), sustained visual attention, visuomotor accuracy, visuomotor speed, symbol digit, visual memory and serial digit learning tests. Significant impairment found in sustained visual attention and symbol digit tests in the cases compared to nonexposed reference $(38,39)$.

A study done on volunteers with varying degrees of occupational exposure to OP showed significant impairment on the Bender Visual Motor Gestalt test (used to evaluate visual motor maturity) and part B of the Trail Making test (Trail Making test is neuropsychological test of visual attention (37).

The study done by Fiedler N et al (1997) recruiting tree fruit farmers who had occupational exposure to OP demonstrated significantly slow simple reaction time for the dominant and nondominent hands of the exposed than the controls. No significant impairment seen in the visuomotor coordination, verbal memory, verbal ability and expressive and receptive language $(38,39)$. 
The review article by Kamel $\mathrm{F}$ et al. (2004) looked into association of pesticide exposure with neurological dysfunction. They found inconsistencies among the studies. Most studies found deficits in one or more tests of cognitive function but different tests were affected in different studies (40).

The current study did not showed persistent impairment of cognitive function following acute OP exposure. The methods we used may not be sensitive enough to detect mild cognitive impairment. Further studies are required with more sophisticated neurobehavioural tests to assess cognitive function following acute OP poisoning.

\section{Acknowledgement}

The studies were funded by the South Asian Clinical Toxicology Research Collaboration, which was funded through the Welcome Trust / NHMRC Australia. I would like to acknowledge participants, the consultants who gave their patients for the study, consultant radiologists and the staff under them, administrative staff and health care professionals at the Teaching Hospital, Karapitiya and the General Hospital, Matara, Heads and the staff members of the Departments of Pharmacology, Medicine, Parasitology, Microbiology and Pathology, Faculty of Medicine, University of Ruhuna, the members and the Clinical Research Assistance of the South Asian Clinical Toxicology Research Collaboration. A special word of thank is extended to Professor A H Dawson, Professor N Buckley, Professor A Fernando, Professor K D Pathirana, Professor P L Ariyananda and Professor B Perera.

\section{References}

1. Stephens R, et al. Neuropsychological effects of long-term exposure to organophosphates in sheep dip. Lancet, 1995; 345(8958): 1135-9.

2. Jayarathnam J. Acute pesticide poisoning: A major global health problem. World Health Statistics Quarterly, 1990; 43: $139-44$.

3. Jayarathnam J, Senevirathna RSDA, and Copplestone JF. Survey of pesticide poisoning in Sri Lanka. Bulletin of the World Health Organization, 1982. 60(4): 615-9.
4. Annual Health Bulletin. Morbidity and Mortality. 2003, Health Ministry Sri Lanka. 21-30.

5. Singh S and Sharma N. Neurological syndromes following organophosphate poisoning. Neurol India, 2000; 48(4): 30813.

6. Ewing DJ, Testing for autonomic neuropathy. Lancet, 1981; 1(8213): 224.

7. Ewing DJ, et al. Cardiac autonomic neuropathy in diabetes: comparison of measures of $\mathrm{R}-\mathrm{R}$ interval variation. Diabetologia, 1981; 21(1): 18-24.

8. Hilz MJ and Dutsch M. Quantitative studies of autonomic function. Muscle Nerve, 2006;33(1): 6-20.

9. Ewing DJ and Clarke BF. Diagnosis and management of diabetic autonomic neuropathy. $\mathrm{Br}$ Med J (Clin Res Ed), 1982; 285(6346): 916-8.

10 Gerawarapong C. Association of peripheral autonomic neuropathy and sympathetic skin response in the patients with diabetic polyneuropathy: A pilot study in Thailand, 2015 Dec; 98(12): 1222-30.

11. Kelly CE. Evaluation of voiding dysfunction and measurement of bladder volume. Rev Urol, 2004; 6(Suppl 1): $\mathrm{S} 32-7$.

12. Guidelines EBM. Determine the volume of residual urine by ultrasonography. EBM Guidelines, 2004.

13. Misra UK, et al. A study of nerve conduction velocity, late responses and neuromuscular synapse functions in organophosphate workers in India. Arch Toxicol, 1988; 61(6): 496-500.

14. Engel LS, et al. Neurophysiological function in farm workers exposed to organophosphate pesticides. Arch Environ Health, 1998; 53(1): 7-14.

15. Aminoff MJ. Electrodiagnosis in clinical Neurology. $5^{\text {th }}$ ed Eelectromyography, nerve conduction studies, and related techniques, ed. A. M.J. Vol. 1. 2005, USA: Elsevier. 231425.

16. Otto D, et al. Electrophysiological measures of visual and auditory function as indices of neurotoxicity. Toxicology, 1988; 49(2-3): 205-18.

17. Legatt AD, Arezzo JC, and Vaughan HG. The anatomic and physiologic bases of brain stem auditory evoked potentials. Neurol Clin, 1988; 6(4): 681-704.

18. De Silva HA and Gunatilake SB. Mini Mental State Examination in Sinhalese: a sensitive test to screen for dementia in Sri Lanka. Int J Geriatr Psychiatry, 2002; 17(2): 134-9. 
19. Worek F, et al. Improved determination of acetylcholinesterase activity in human whole blood. Clin Chim Acta, 1999; 288(1-2): 73-90.

20. Jayasinghe SS, Pathirana KD, and Buckley NA. Effects of acute organophosphorus poisoning on function of peripheral nerves: a cohort study. PLoS One, 2012; 7(11): e49405.

21. Jayasinghe SS and Pathirana KD. Autonomic function following acute organophosphorus poisoning: a cohort study. PLoS One, 2012; 7(5): e37987.

22. Jayasinghe SS and Pathirana KD. Effects of deliberate ingestion of organophosphate or paraquat on brain stem auditory-evoked potentials. J Med Toxicol, 2011; 7(4): 27780.

23. Jayasinghe SS. Effects of acute organophosphate ingestion on cognitive function, assessed with the mini mental state examination. J Postgrad Med, 2012; 58(3): 171-5.

24. Ruijten MW, et al. Effect of chronic mixed pesticide exposure on peripheral and autonomic nerve function. Arch Environ Health, 1994; 49(3): 188-95.

25. Stalberg E. Neuromuscular transmission studied with single fibre electromyography. Acta Anaesthesiol Scand Suppl, 1978; 70: 112-7.

26. Sanders DB, Stalberg EV. Single-fiber electromyography. Muscle Nerve, 1996; 19(9): 1069-83.

27. Jayawardane $\mathrm{P}$, et al. The spectrum of intermediate syndrome following acute organophosphate poisoning: a prospective cohort study from Sri Lanka. PLoS Med, 2008; 5(7): e147.

28. Stalberg E, et al. Effect of occupational exposure to organophosphorus insecticides on neuromuscular function. Scand J Work Environ Health, 1978; 4(3): 255-61.

29. Yang D, He F, and Li T. Repetitive nerve stimulation and stimulation single fiber electromyography studies in rats intoxicated with single or mixed insecticides. Toxicology, 2001; 161(1-2):111-6.

30. Dongren Y, Tao L, and Fengsheng H. Electroneurophysiological studies in rats of acute dimethoate poisoning. Toxicol Lett, 1999; 107(1-3): 249-54.
31. Maselli RA, Soliven BC. Analysis of the organophosphateinduced electromyographic response to repetitive nerve stimulation: paradoxical response to edrophonium and D-tubocurarine. Muscle Nerve, 1991; 14(12): 1182-8.

32. Jayawardane P, Senanayake N, and Dawson A. Electrophysiological correlates of intermediate syndrome following acute organophosphate poisoning. Clin Toxicol (Phila), 2009; 47(3): 193-205.

33. Bielefeld EC, et al. Damage and threshold shift resulting from cochlear exposure to paraquat-generated superoxide. Hear Res, 2005; 207(1-2): 35-42.

34. Harris $\mathrm{KC}$, et al. Increased resistance to free radical damage induced by low-level sound conditioning. Hear Res, 2006; 213(1-2): 118-29.

35. Clerici WJ and Yang L. Direct effects of intraperilymphatic reactive oxygen species generation on cochlear function. HearRes, 1996; 101(1-2): 14-22.

36. Kimura K, et al. Effects of pesticides on the peripheral and central nervous system in tobacco farmers in Malaysia: studies on peripheral nerve conduction, brain-evoked potentials and computerized posturography. Ind Health, 2005. 43(2): 285-94.

37. Korsak RJ, MM Sato. Effects of chronic organophosphate pesticide exposure on the central nervous system. Clin Toxicol, 1977; 11(1): p83-95.

38. Steenland $\mathrm{K}$, et al. Chronic neurological sequelae to organophosphate pesticide poisoning. Am J Public Health, 1994; 84(5): 731-6.

39. Fiedler $\mathrm{N}$, et al. Long-term use of organophosphates and neuropsychological performance. Am J Ind Med, 1997; 32(5): 487-96.

40. Kamel F and Hoppin JA. Association of pesticide exposure with neurologic dysfunction and disease. Environ Health Perspect, 2004; 112(9): 950-8. 